\title{
Natural convection flow of air in an inclined open cavity
}

\author{
M. Nateghi* $\quad$ S. W. Armfield ${ }^{\dagger}$ \\ (Received 8 August 2003; revised 27 January 2004)
}

\begin{abstract}
Consider natural convection flow in a two dimensional inclined open cavity for both transient and steady-state flow. The left hand vertical wall of cavity is heated and facing an opening. The top and bottom boundaries are insulated. Numerical solutions are obtained for the Navier-Stokes equations on a non-staggered grid using a finite volume scheme. Results are considered for a wide range of Rayleigh numbers varying from $10^{5}$ to $10^{10}$ with Prandtl number 0.7 and inclination angles from $10^{\circ}$ to $90^{\circ}$. The flow is steady at the low Rayleigh number for all angles and becomes unsteady at the high Rayleigh number for all angles. The critical Rayleigh numbers for all angles are obtained and we show that the critical Rayleigh number decreases as the inclination angle increases.
\end{abstract}

*School of Aerospace, Mechanical \& Mechatronic Engineering, University of Sydney, Sydney, Australia. mailto:morteza@aeromech.usyd.edu.au

†mailto: armfield@aeromech.usyd.edu.au

See http://anziamj.austms.org.au/V45/CTAC2003/Nate/home.html for this article, (C) Austral. Mathematical Soc. 2004. Published August 14, 2004. ISSN 1446-8735 


\section{Contents}

1 Introduction

C871

2 Numerical method

C872

3 Results and discussion

C874

3.1 Low Rayleigh number and steady state flow . . . . . . . . C874

3.2 High Rayleigh number and unsteady flow . . . . . . . . C C877

4 Conclusions

C888

References

C889

\section{Introduction}

Natural convection in a cavity with one vertical side open has a wide range of industrial applications and embodies much of the fundamental fluid mechanics of the more commonly studied enclosed cavity flow. Some applications are solar thermal receiver systems [8], fire research, electronic cooling [6], and energy-saving household refrigerators. Chan and Tien [7] carried out an experimental and numerical analysis of a side opening cavity with a length to height aspect ratio of 0.143 for Rayleigh numbers ranging from $10^{6}$ to $10^{7}$. They found that the rate of heat transfer approached that for a vertical heated flat plate, which has a similar flow next to the heated wall. In the present study we investigate natural convection in a two dimensional open cavity for both transient and steady-state flow. The cavity has one vertical heated wall facing an open wall, with the other two walls insulated. Numerical solutions are obtained for the Navier-Stokes equations on a non-staggered grid using a finite volume scheme. Results are considered for Rayleigh numbers varying from $10^{5}$ to $10^{10}$. The Prandtl number is set to 0.7 (air) for all calculations and the inclination angle is varied in the range of $10^{\circ}$ to $90^{\circ}$. 


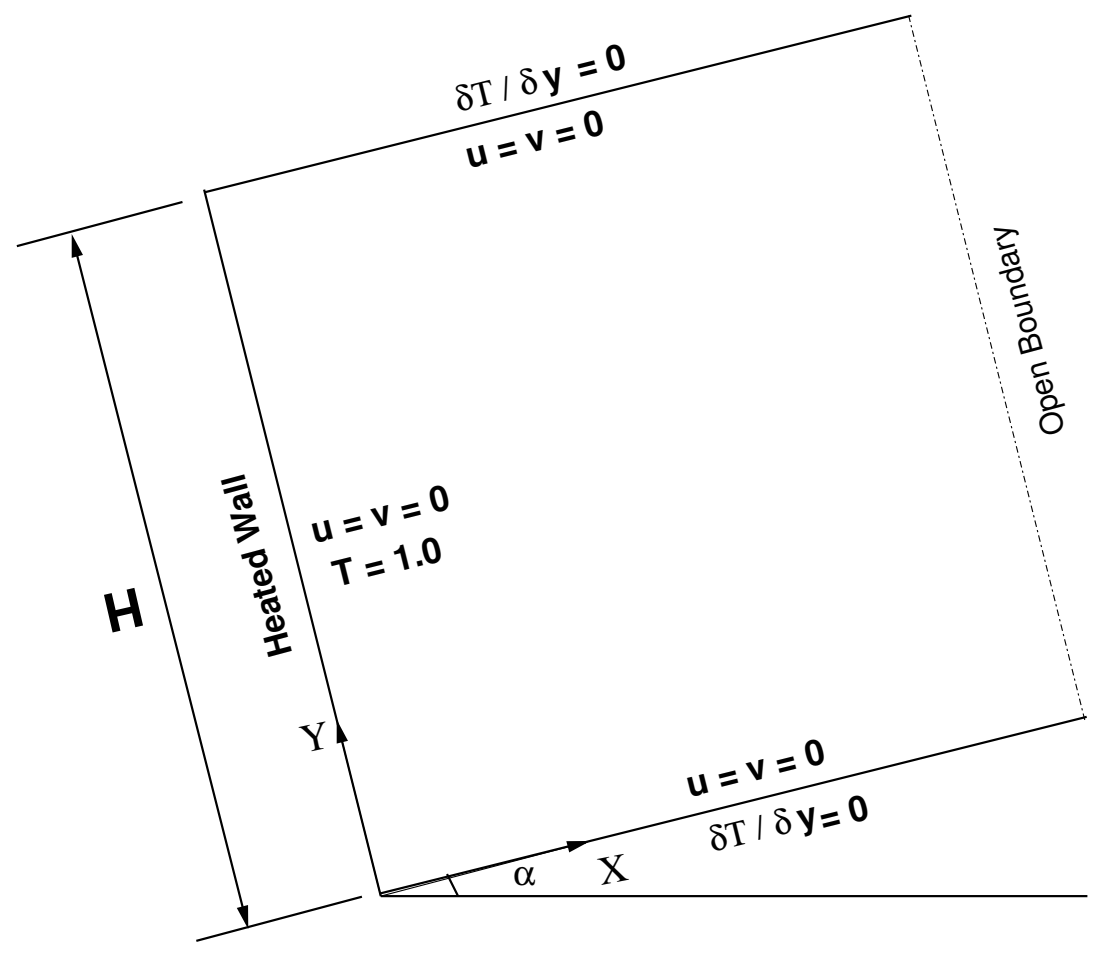

Figure 1: Geometry and boundary condition.

\section{$2 \quad$ Numerical method}

The flow is described by the Navier-Stokes equations and the temperature equation, with the Boussinesq assumption to use their incompressible form. The equations are written in conservative, non-dimensional form in Cartesian coordinates:

$$
\begin{gathered}
\frac{\partial u}{\partial x}+\frac{\partial v}{\partial y}=0 \\
\frac{\partial u}{\partial t}+\frac{\partial(u u)}{\partial x}+\frac{\partial(v u)}{\partial y}=-\frac{\partial p}{\partial x}+\left(\frac{\partial^{2} u}{\partial x^{2}}+\frac{\partial^{2} u}{\partial y^{2}}\right)+\frac{\operatorname{Ra}}{\operatorname{Pr}} T \sin \alpha
\end{gathered}
$$




$$
\begin{gathered}
\frac{\partial v}{\partial t}+\frac{\partial(u v)}{\partial x}+\frac{\partial(v v)}{\partial y}=-\frac{\partial p}{\partial y}+\left(\frac{\partial^{2} v}{\partial x^{2}}+\frac{\partial^{2} v}{\partial y^{2}}\right)+\frac{\operatorname{Ra}}{\operatorname{Pr}} T \cos \alpha \\
\frac{\partial T}{\partial t}+\frac{\partial(u T)}{\partial x}+\frac{\partial(v T)}{\partial y}=\frac{1}{\operatorname{Pr}}\left(\frac{\partial^{2} T}{\partial x^{2}}+\frac{\partial^{2} T}{\partial y^{2}}\right)
\end{gathered}
$$

The Rayleigh number and Prandtl number are

$$
\mathrm{Ra}=\frac{g \beta \Delta T H^{3}}{\nu \kappa}
$$

and

$$
\operatorname{Pr}=\frac{\nu}{\kappa},
$$

respectively, where $g$ is the acceleration due to gravity, $\beta$ is the coefficient of thermal expansion, $\Delta T$ is the total temperature variation, $\nu$ is the kinematic viscosity, $H$ is cavity height, $t$ is the time, $\alpha$ is the inclination angle, $T$ is temperature, $\kappa$ is the thermal diffusivity, $u$ is the velocity component in the $x$-direction and $v$ is the velocity component in the $y$-direction. Figure 1 shows the geometry of the open cavity, the coordinate system and boundary conditions for the present study. On the open boundary the $x$ derivations of the velocities are set to zero, while for $u$-velocity positive $\partial T / \partial x=0$ and $u$ velocity negative $T=0$. Initially the fluid is at rest and isothermal $(T=0)$ and at time $t=0$ the left side wall is instantaneously heated to $T=1.0$.

The advective terms are approximated using QUICK scheme [10]. The second-order Adams-Bashforth scheme and Crank-Nicholson scheme are used for the time integration of the advective terms and the diffusive terms, respectively. The momentum and temperature equations are solved using an ADI scheme. To enforce the continuity, the pressure correction method is used to construct a Poisson's equation which is solved using the preconditioned GMRES method. Detailed descriptions of these schemes were given by Armfield $[3,5]$ and the code has been previously used and validated for the simulation of a range of buoyancy dominated flows $[11,1,2,9]$. A nonuniform mesh is used allowing grid nodes to be concentrated in the region of 
the heated wall and the upper and lower boundaries. The origin is located at the bottom left corner of the domain with y increasing up the hot wall and $x$ increasing horizontally into the domain. The horizontal mesh size adjacent to the hot wall is $\Delta w_{x}=0.001$ with a grid stretching factor of 1.07 per cell unit until $x=0.1$, the stretching factor is then gradually reduced until the grid become uniform in the interior. The vertical mesh size adjacent to the insulated boundaries is $\Delta w_{y}=0.001$ with the same stretching factor per cell unit until $y=0.1$ for the lower domain and $y=0.9$ for the upper domain. The grid stretching factor is then gradually reduced until the grid becomes uniform in the interior. This gives a basic grid of $101 \times 129$ nodes which is used with a basic time step of $\Delta t=5.0 \times 10^{-7}$. Mesh and time step dependence tests have been carried out by comparing the solution obtained on the basic mesh of $101 \times 129$ and two more meshes of $67 \times 87$ and $215 \times 259$ nodes. The comparison between the three mesh sizes results show very small variations, indicating that the basic mesh and time step provide sufficient resolution. In the present study the mesh of $101 \times 129$ nodes is selected to get more accurate solutions.

\section{Results and discussion}

Results are presented for Rayleigh numbers varying from $\mathrm{Ra}=10^{5}$ to $\mathrm{Ra}=$ $5 \times 10^{10}$ with the inclination angles of $\alpha=10^{\circ}, 30^{\circ}, 60^{\circ}$ and $90^{\circ}$.

\subsection{Low Rayleigh number and steady state flow}

Figures 2 and 3 present the fully developed stream-function and temperature contours respectively for $\mathrm{Ra}=10^{5}$ with inclination angles of $\alpha=10^{\circ}, 30^{\circ}$, $60^{\circ}$ and $90^{\circ}$ with hot wall on the left and open boundary on the right at time $t=0.2$ for all angles except $\alpha=90^{\circ}$ which has a larger development time and is shown at $t=0.605$. At full development the flow is steady state 
10 degree angle

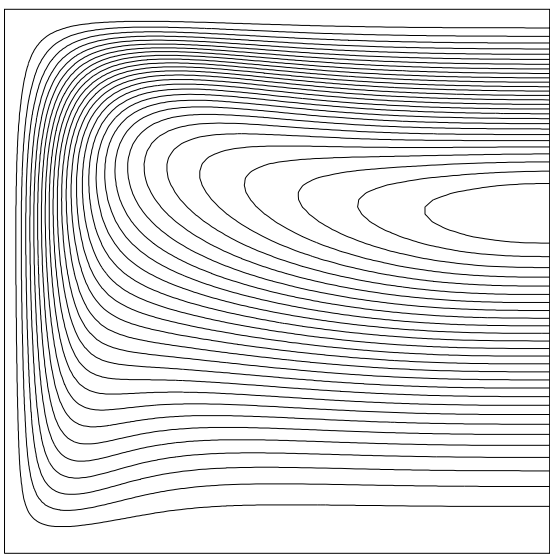

60 degree angle

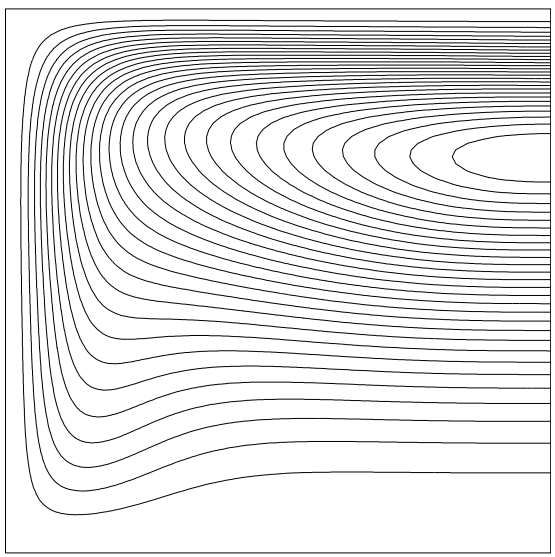

30 degree angle

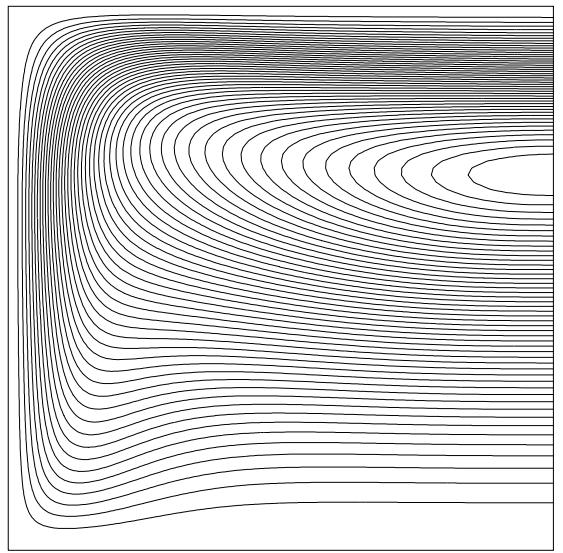

90 degree angle

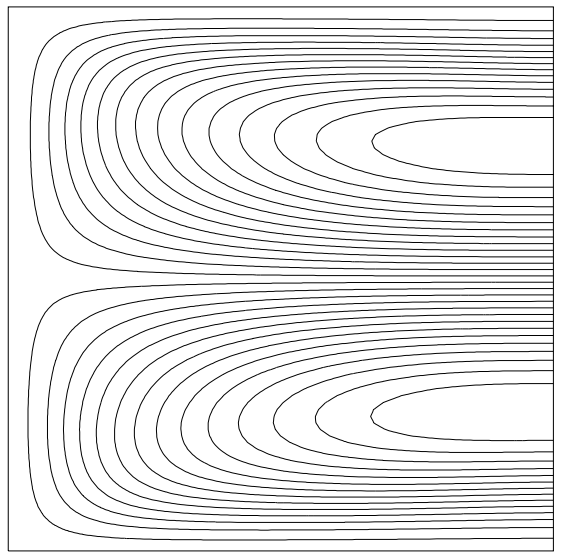

(a)

Figure 2: Streamline contours of the flow for Ra $=10^{5}$. 


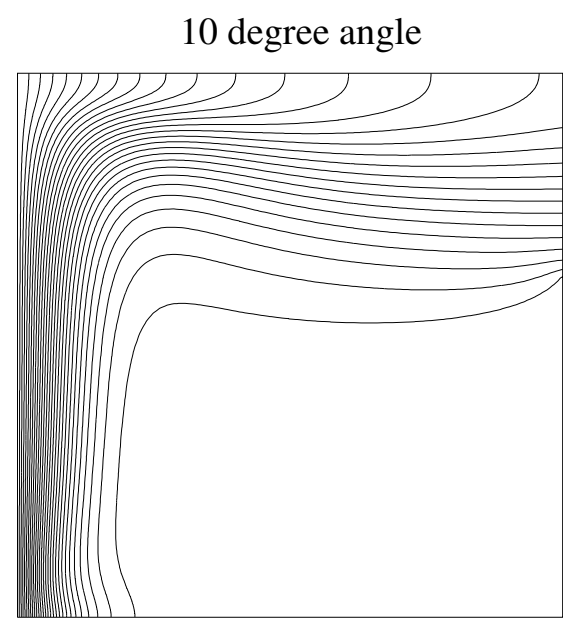

60 degree angle

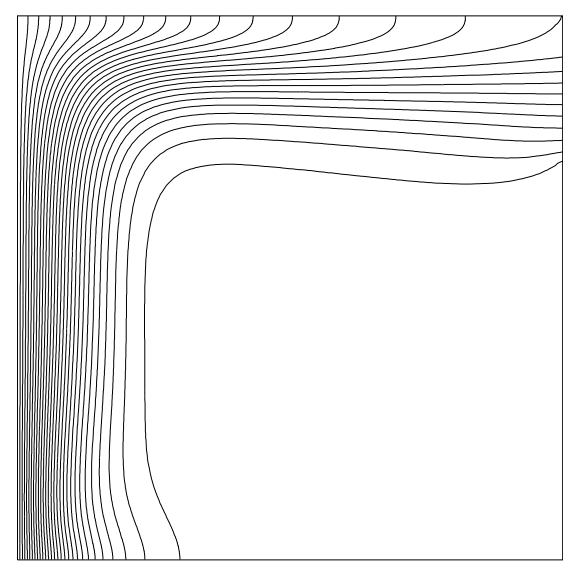

30 degree angle

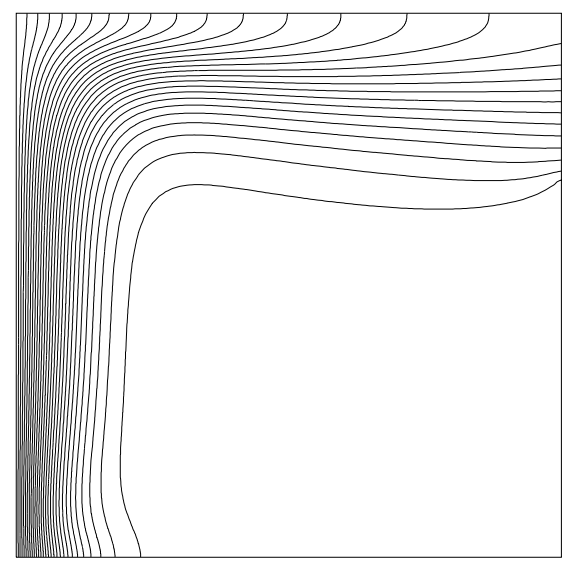

90 degree angle

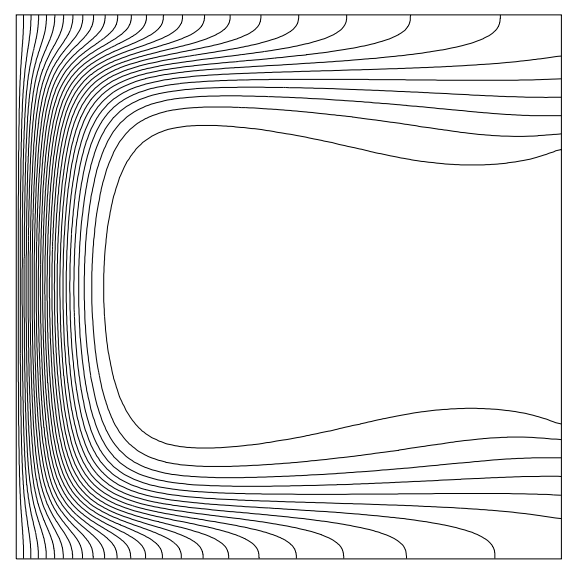

(b)

Figure 3: Temperature contours of the flow for $\mathrm{Ra}=10^{5}$. 
for all $\alpha$ at this Rayleigh number, as expected based on the critical Rayleigh number for the convective instability of a natural convection boundary layer as reported by Armfield and Janssen [4]. For the inclination angle of $\alpha=10^{\circ}$ the flow enters cavity from the lower half of the open boundary and exits the cavity through the upper part of the open boundary. For the inclination angles of $\alpha=30^{\circ}$ and $60^{\circ}$ the basic flow structure is the same but with the discharged fluid occupying a smaller part of the upper region of the cavity. For inclination angle $\alpha=90^{\circ}$ the fluid enters the cavity from the centre of the open boundary and leaves at the outer regions, forming two circulations appearing identical in size.

See in Figure 3 that the isotherms show a thick thermal boundary layer is formed on the heated wall, and also a thick hot intrusion on the upper insulated wall forms for all angles except $\alpha=90^{\circ}$ which shows a quite different structure. The thickness of the thermal boundary layer increases with increasing inclination angle from $\alpha=10^{\circ}$ to $60^{\circ}$ but for $\alpha=90^{\circ}$ the thickness of the thermal boundary layer is small at the centre and becomes thicker at the lower and upper part of the boundary layer. The thickness of the upper hot intrusion at the open boundary decreases from $\alpha=10^{\circ}$ to $60^{\circ}$ except for $\alpha=90^{\circ}$. For $\alpha=90^{\circ}$ the upper and lower intrusions are formed on the insulated boundaries and the sizes of the intrusions appears almost identical. For this study the flow adjacent to the insulated wall has been defined as an intrusion for all angles for simplicity. The temperature time series adjacent to the hot wall for $\mathrm{Ra}=10^{5}$ with the inclination angles of $\alpha=10^{\circ}, 30^{\circ}$, $60^{\circ}$ and $90^{\circ}$ confirm that at this Rayleigh number the flow is steady state for all angles.

\subsection{High Rayleigh number and unsteady flow}

$\boldsymbol{\alpha}=10^{\circ}$ flow: Figure 4 shows the fully developed stream-function contours, temperature contours, and temperature time series adjacent to the hot wall for $\mathrm{Ra}=10^{9}$ with $\alpha=10^{\circ}$ at $t=0.19$. Although this flow is also steady, 

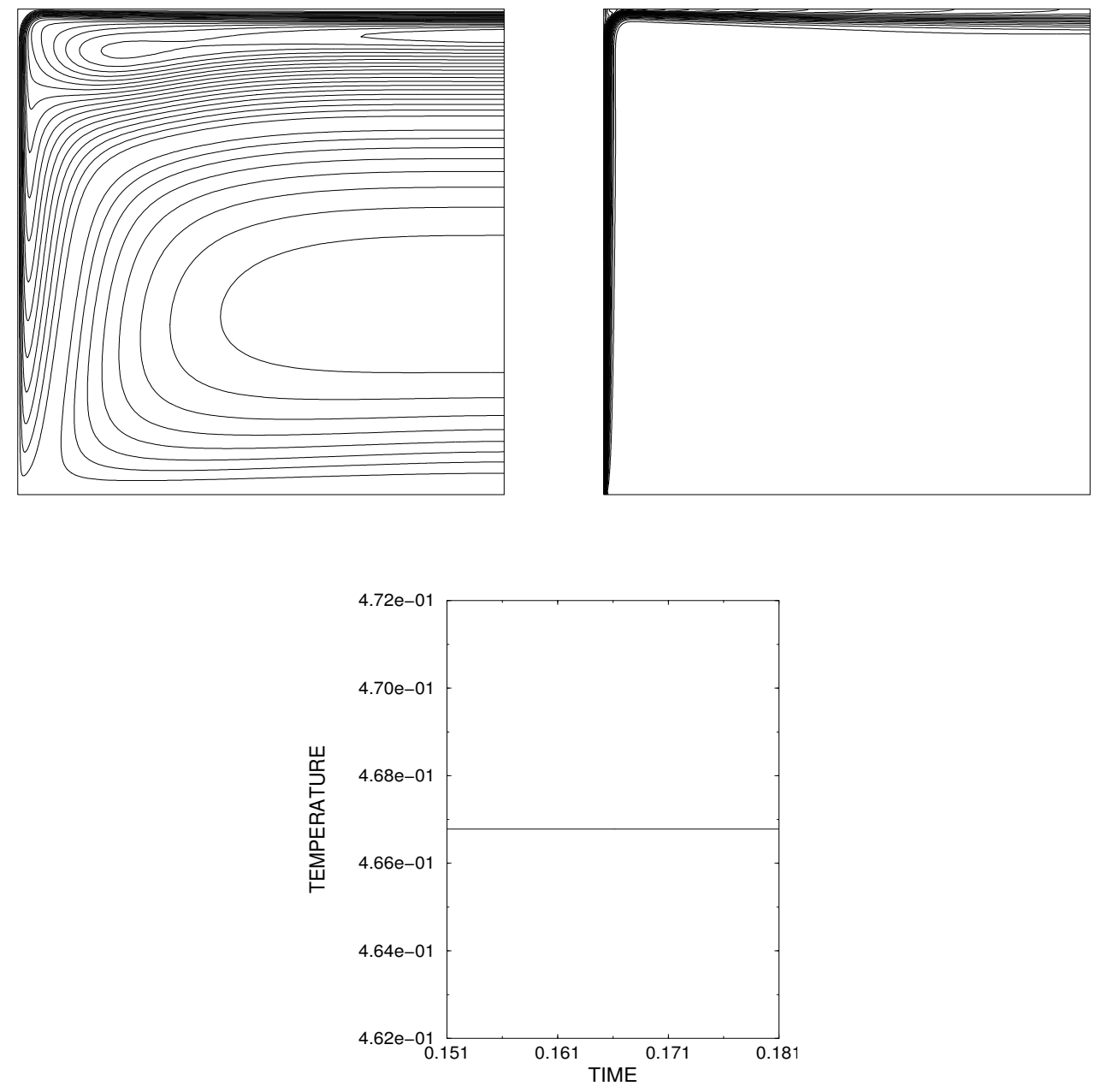

(a)

Figure 4: Streamline and temperature contours of $\alpha=10^{\circ}$ flow with temperature time series at $\mathrm{Ra}=10^{9}$. 

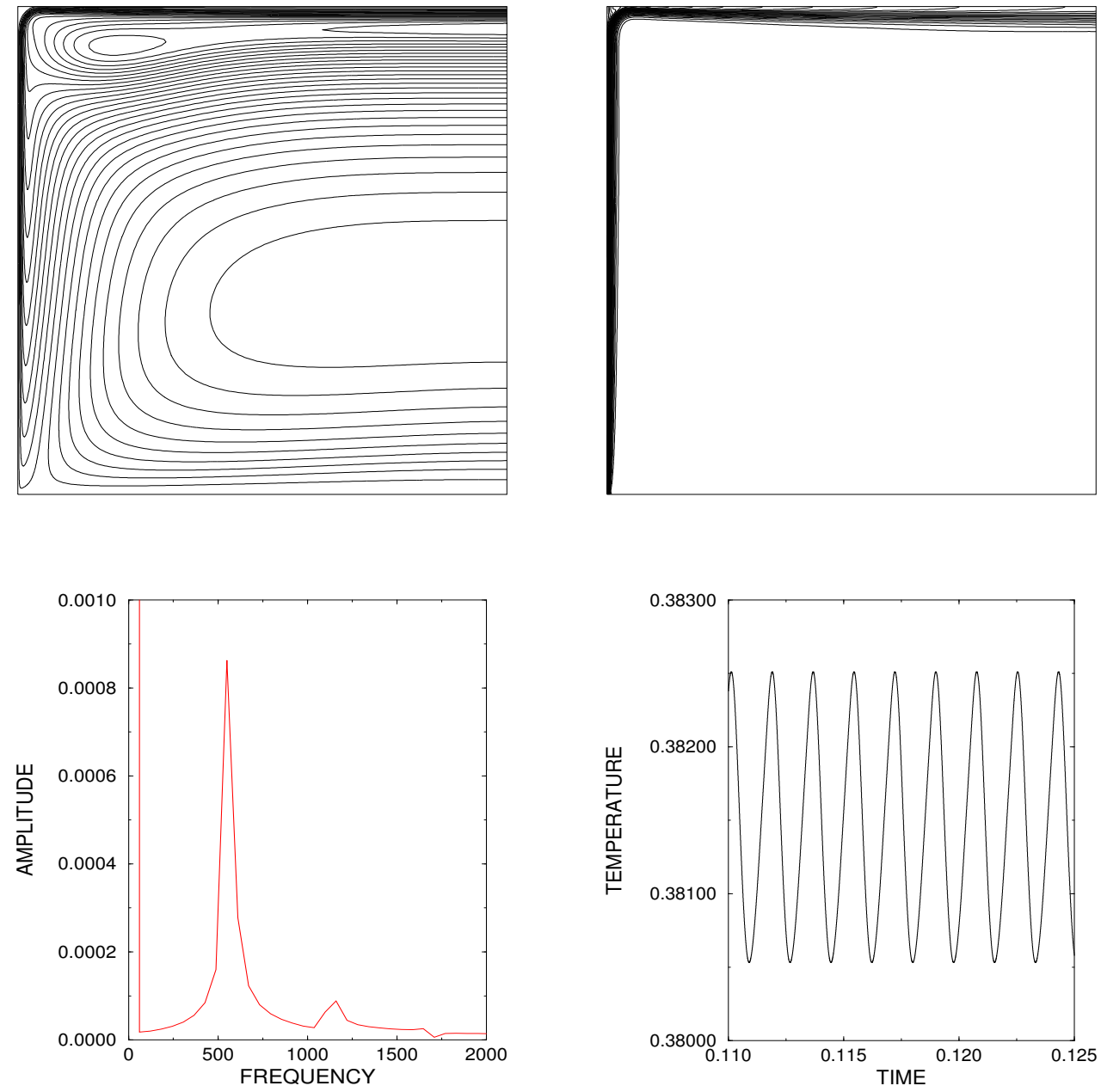

(b)

Figure 5: Streamline and temperature contours of $\alpha=10^{\circ}$ flow with temperature time series and the associated spectra at $\mathrm{Ra}=2 \times 10^{9}$. 
the flow structure is different to that observed for the low Rayleigh number. The flow enters the cavity from the upper half of the open boundary and leaves the cavity at the outer regions of the open boundary, forming two circulations. The thermal boundary layer is thiner than that observed for the lower Rayleigh number, which is expected as the boundary layer thickness scales as $\delta_{T}=H / \mathrm{Ra}^{1 / 4}$.

Figure 5 presents the fully developed stream-function contours, temperature contours, temperature time series and spectra for a point adjacent to the hot wall for $\mathrm{Ra}=2 \times 10^{9}$ again with $\alpha=10^{\circ}$ at $t=0.19$. The flow structure is similar to $\mathrm{Ra}=10^{9}$ flow with only a small difference in the relative size of the two circulations. However, see in the temperature time series that the flow is now unsteady; it is periodic. The frequency of this signal, obtained using Fourier transform, is determined from the spectra to be $f=549$. The flow is then supercritical for this Rayleigh number and thus the critical Rayleigh number lies between $10^{9}$ and $2 \times 10^{9}$.

$\boldsymbol{\alpha}=30^{\circ}$ flow: Figure 6 presents the fully developed stream-function contours, temperature contours and temperature time series adjacent to the hot wall for $\mathrm{Ra}=4 \times 10^{8}$ with $\alpha=30^{\circ}$ at $t=0.128$. The flow is steady with a structure quite similar to that observed for $\mathrm{Ra}=10^{9}$ with $\alpha=10^{\circ}$ with only a small difference in the relative size of the two circulations and in the boundary layer and intrusions thickness. Figure 7 presents the fully developed stream-function contours, temperature contours, temperature of time series and spectra at a point adjacent to the hot wall for $\mathrm{Ra}=5 \times 10^{8}$ with $\alpha=30^{\circ}$ at $t=0.125$. The flow structure is similar to the $\mathrm{Ra}=4 \times 10^{8}$ flow with again only a small difference in the relative size of the two circulations. The temperature time series indicate the flow is unsteady with a periodic behavior. The frequency of this signal, obtained using the Fourier transform, is determined to be $f=457$. The $\alpha=30^{\circ}$ flow at $\mathrm{Ra}=5 \times 10^{8}$ is defined as supercritical and the critical Rayleigh number then lies between $4 \times 10^{8}$ and $5 \times 10^{8}$. 

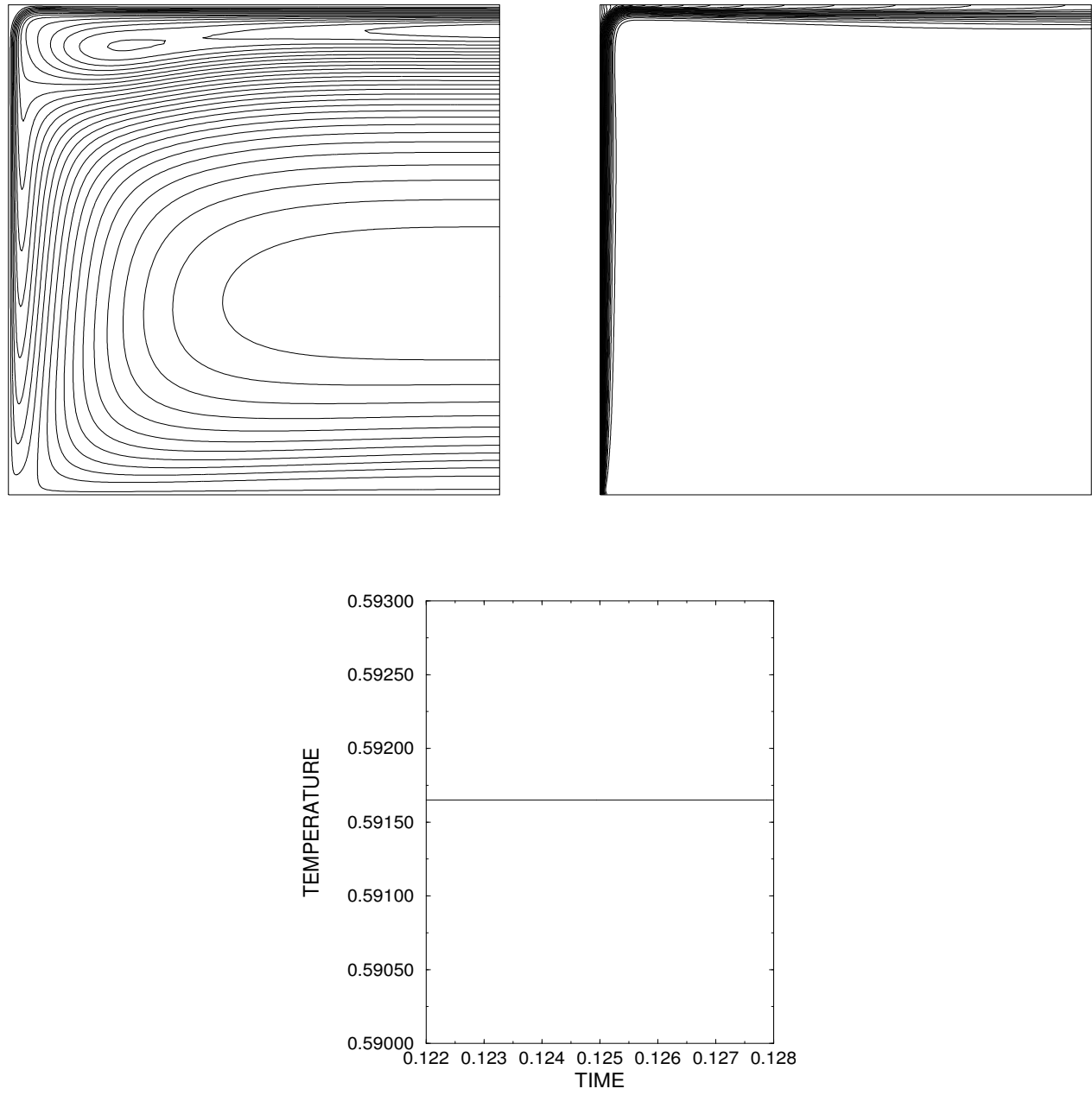

(a)

Figure 6: Streamline and temperature contours of $\alpha=30^{\circ}$ flow with temperature time series at $\mathrm{Ra}=4 \times 10^{8}$. 

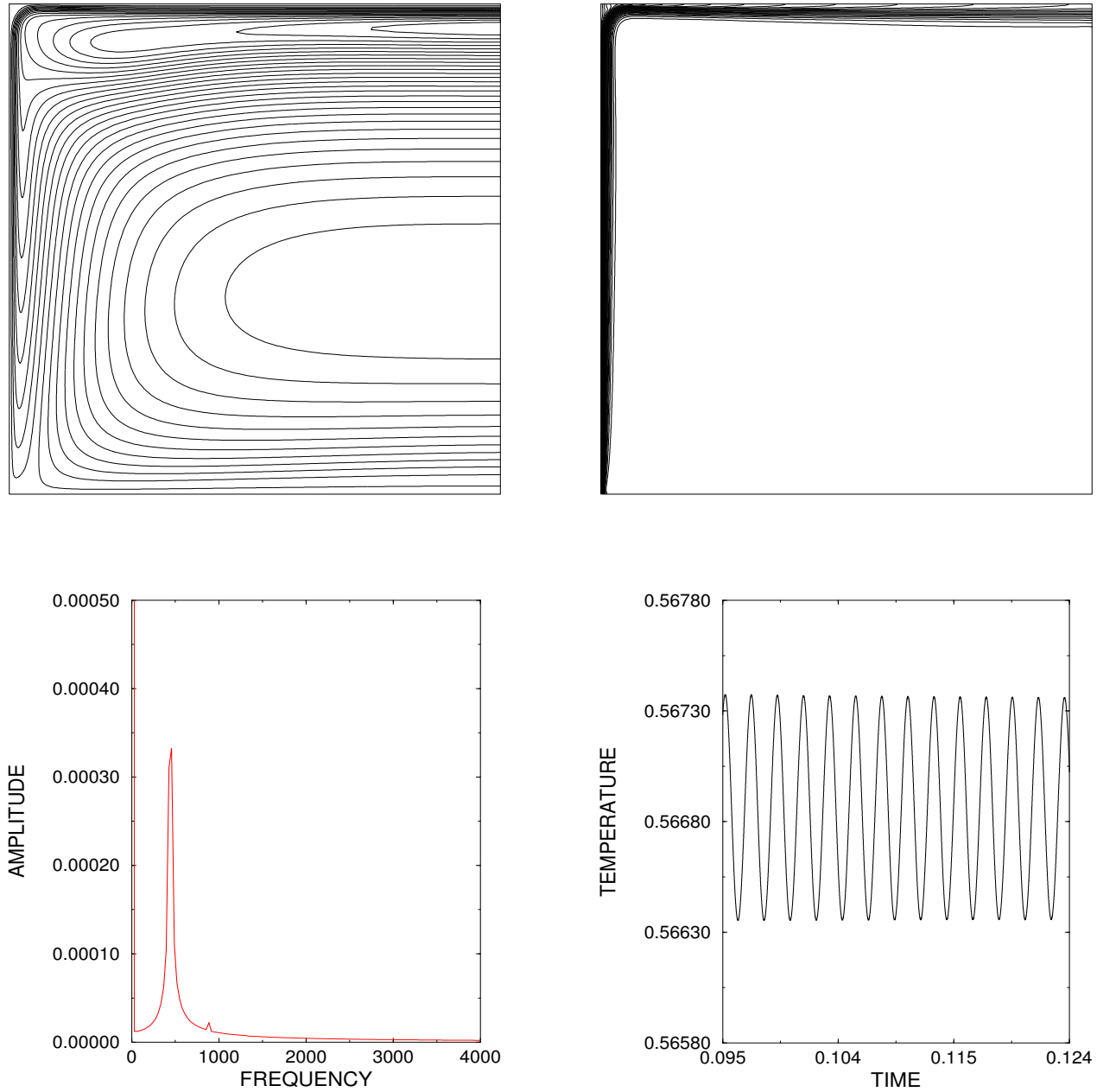

(b)

Figure 7: Streamline and temperature contours of $\alpha=30^{\circ}$ flow with temperature time series and the associated spectra at $\mathrm{Ra}=5 \times 10^{8}$. 

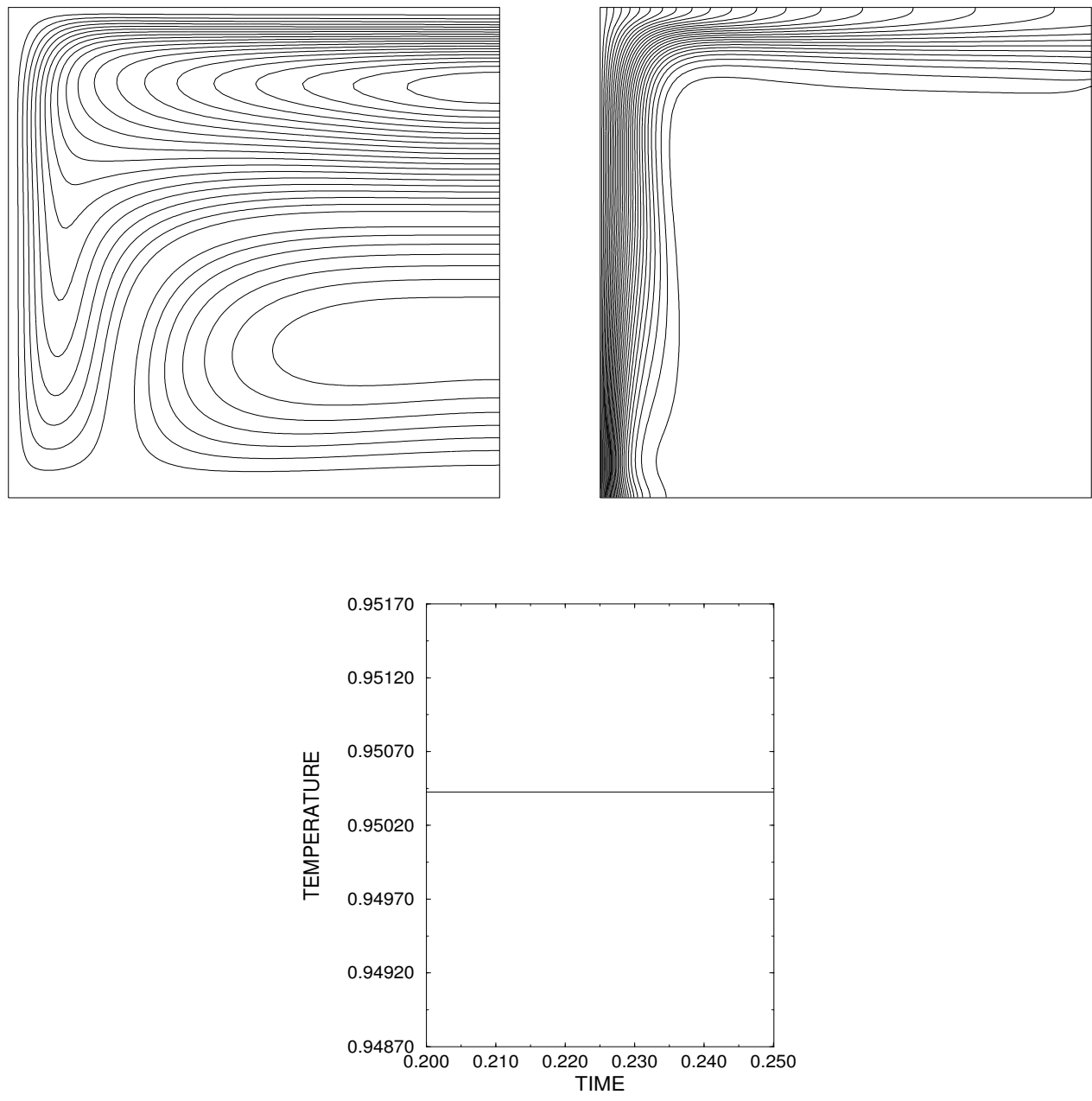

(a)

Figure 8: Streamline and temperature contours of $\alpha=60^{\circ}$ flow with temperature time series at $\mathrm{Ra}=10^{6}$. 

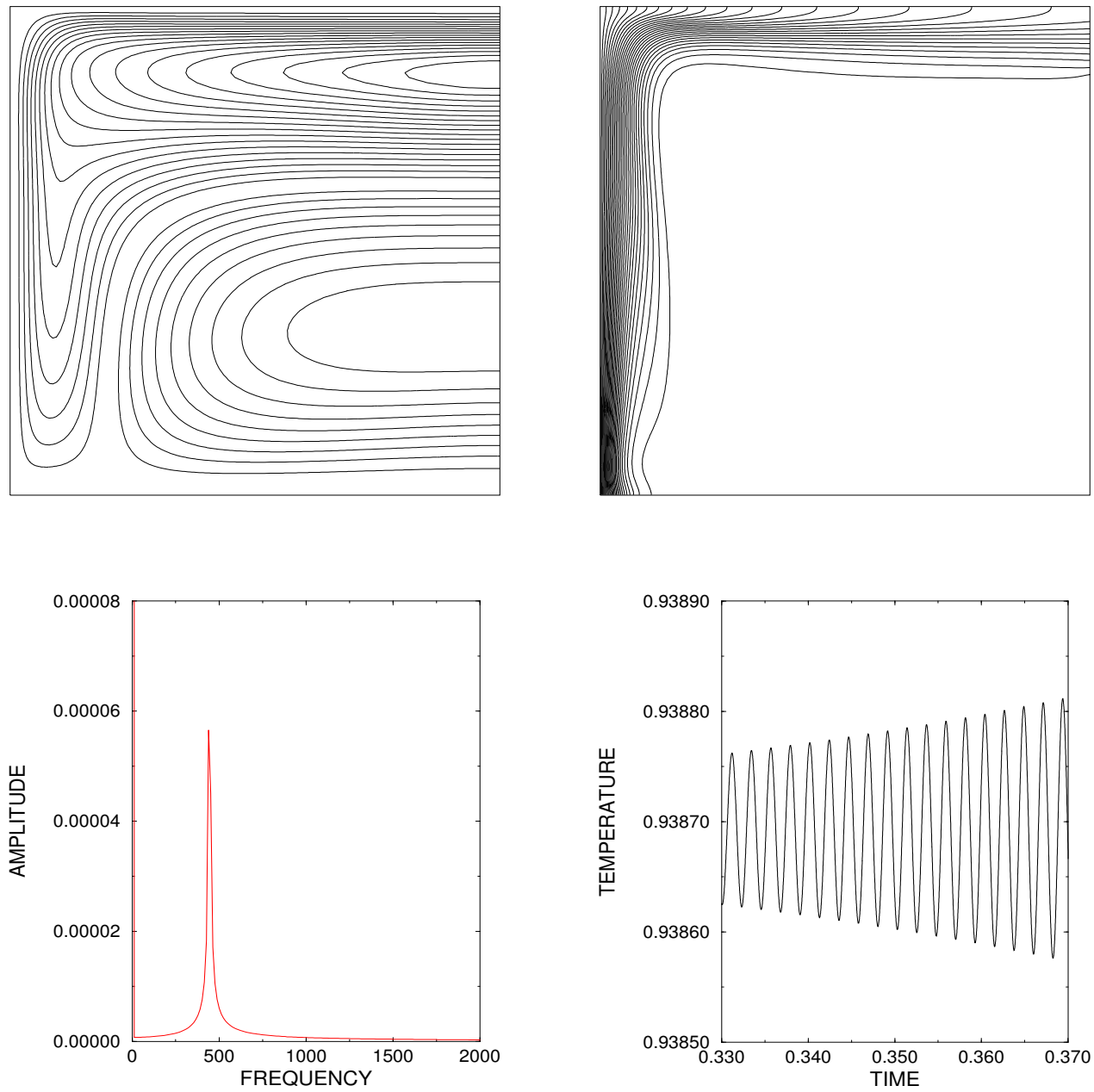

(b)

Figure 9: Streamline and temperature contours of $\alpha=60^{\circ}$ flow with temperature time series and the associated spectra at $\mathrm{Ra}=2 \times 10^{6}$. 
$\alpha=60^{\circ}$ flow: Figure 8 presents the fully developed stream-function contours, temperature contours and temperature time series adjacent to the hot wall for $\mathrm{Ra}=10^{6}$ with $\alpha=60^{\circ}$ at $t=0.29$. The flow structure is quite different to that observed for the low Rayleigh number flow for this angle. The flow enters cavity from the centre of the open boundary and leaves the cavity from the outer regions forming two circulations. The boundary layer and intrusion thickness is smaller than that observed for the low Rayleigh number flow.

Figure 9 presents the fully developed stream-function contours, temperature contours, temperature time series and spectra at a point adjacent to the hot wall for $\mathrm{Ra}=2 \times 10^{6}$ with $\alpha=60^{\circ}$ at $t=0.37$. The flow structure and isotherms are similar to that observed for $\mathrm{Ra}=10^{6}$ with only a small difference in the relative size of the two circulations and in the boundary layer and intrusions thickness. The temperature time series indicate the flow is unsteady with a quasi-periodic behavior. The frequency of this signal, obtained using the Fourier transform, is determined to be $f=487$. The $\alpha=60^{\circ}$ flow at $\mathrm{Ra}=2 \times 10^{6}$ is defined as supercritical and the critical Rayleigh number then lies between $10^{6}$ and $2 \times 10^{6}$.

$\alpha=90^{\circ}$ flow: Figure 10 demonstrates the fully developed stream-function contours, temperature contours, temperature time series and spectra at a point adjacent to the hot wall for $\mathrm{Ra}=5 \times 10^{5}$ with $\alpha=90^{\circ}$ at $t=0.4$. The flow enters the cavity from the centre and exits the cavity from the outer regions of the open boundary, forming two circulations. The flow structure is quite similar to the low Rayleigh number flow for $\alpha=90^{\circ}$ with a difference of the relative sizes of the two circulations. The isotherm is also quite similar to that observed for the low Rayleigh number but the sizes of the thermal boundary layer and intrusions are smaller. Although the temperature time series show the flow is unsteady, the amplitude of the signal is very low and the signal decaying, thus this flow is classified as sub-critical. Figure 11 presents the fully developed stream-function contours, temperature 

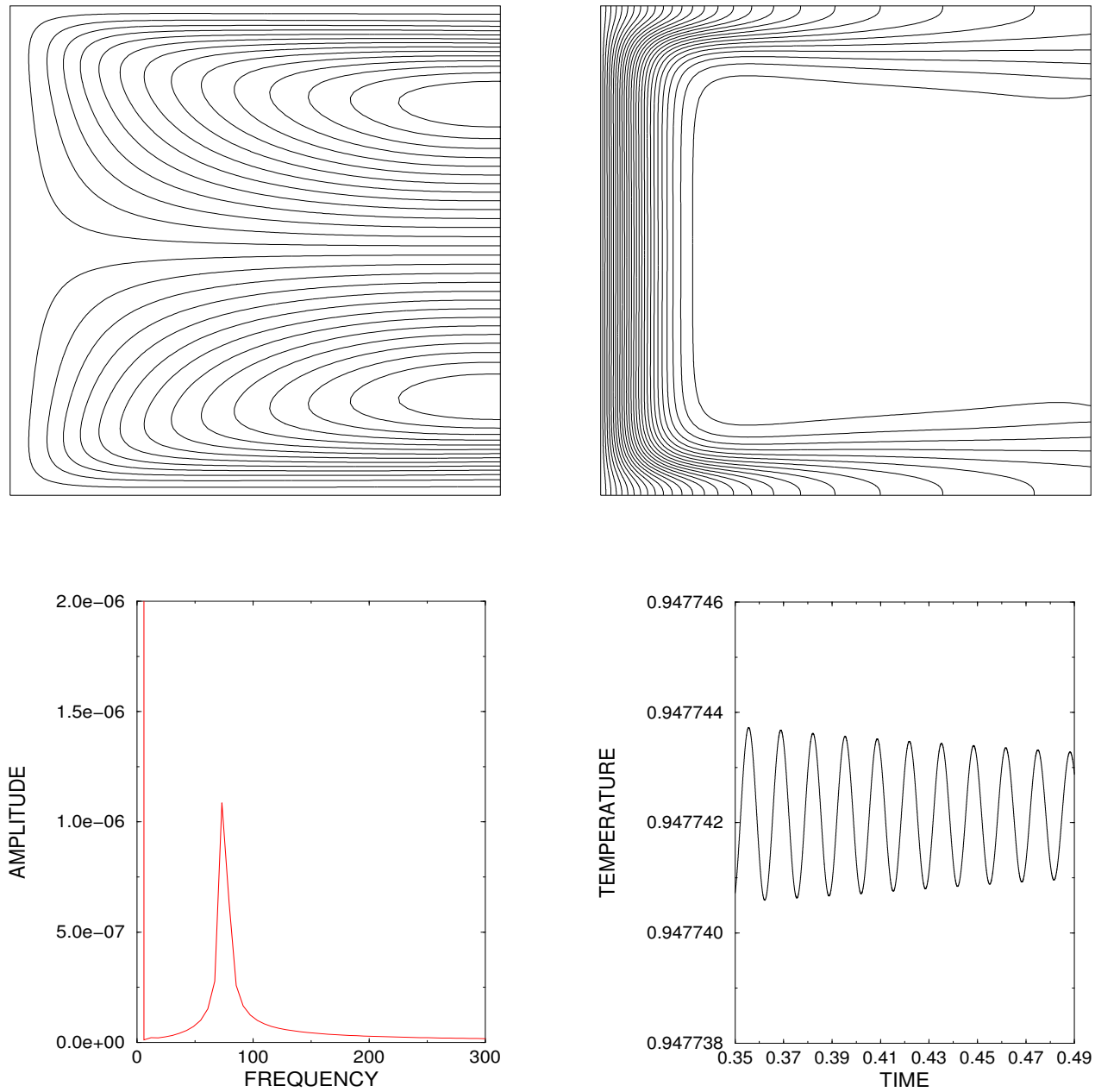

(a)

Figure 10: Streamline and temperature contours (top) and temperature time series with the associated spectra (bottom) for $\alpha=90^{\circ}$ flow at $\mathrm{Ra}=$ $5 \times 10^{5}$. 

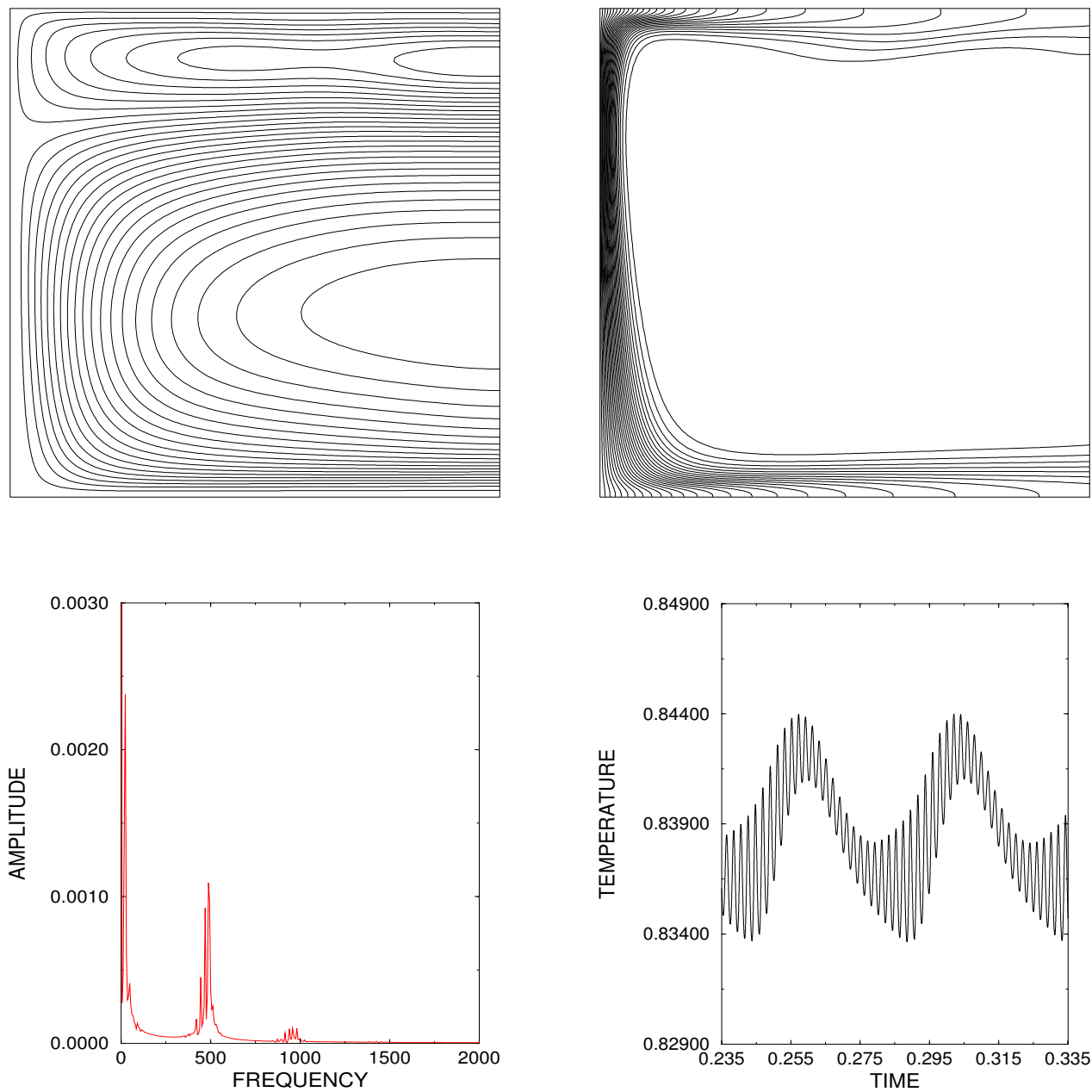

(b)

Figure 11: Streamline and temperature contours (top) and temperature time series with the associated spectra (bottom) for $\alpha=90^{\circ}$ flow at $\mathrm{Ra}=$ $5 \times 10^{6}$. 
contours, and temperature and spectra of time series adjacent to the hot wall for $\mathrm{Ra}=5 \times 10^{6}$ with $\alpha=90^{\circ}$ at $t=0.225$. As is shown in Figure 11 the flow structure is different with that observed for $\mathrm{Ra}=5 \times 10^{5}$ shown in Figure 10. The sizes of the two circulations are unequal, and transient which is associated with the unsteady nature of the flow. The temperature time series show the flow is unsteady with a quasi-periodic behavior. The signal has a dominant frequency and the amplitude of the signal is very much higher than that observed for the lower Rayleigh number indicating that the flow is supercritical, and the critical Rayleigh number for this angle then lies between $5 \times 10^{5}$ and $5 \times 10^{6}$.

\section{Conclusions}

Natural convection flow in an inclined open cavity for Rayleigh numbers varying from $10^{5}$ to $10^{10}$ with Prandtl number 0.7 and the inclination angles from $10^{\circ}$ to $90^{\circ}$ has been investigated. Flow patterns and isotherms are shown in order to give a better understanding of the heat transfer and the flow mechanisms inside the cavity. We observe that the flow is steady for the low Rayleigh number at all angles and becomes unsteady for the high Rayleigh number at all angles. For the high Rayleigh number at all angles the flow forms two circulations. The sizes of the two circulations become almost identical at $\alpha=90^{\circ}$ for the sub-critical Rayleigh number but for the high Rayleigh number with $\alpha=90^{\circ}$ the circulations are unequal, and possess transients which may be associated with the unsteady nature of the flow. At high Rayleigh number for $\alpha=90^{\circ}$ we find that when one of the circulation increases in size, the other shrinks, therefore we suggest that the occurrence of the bifurcation is correlated with the presence of this mechanism and the interaction of these circulations generate the basic instability for this angle. However, for the angles $\alpha=10^{\circ}$ and $30^{\circ}$ at high Rayleigh number, the circulations are also unequal but steady. For the angles $\alpha=10^{\circ}$ and $30^{\circ}$ the ratio between the size of the hot circulation (the flow with streamlines passing 
through the thermal boundary layer) and the size of the cold circulation (the flow with streamlines does not pass through the thermal boundary layer) becomes smaller with increasing Rayleigh number. This double circulation flow structure may be associated with forming the unsteady flow for the angles $\alpha=10^{\circ}$ and $30^{\circ}$. The critical Rayleigh numbers for all angles have been obtained and we showed that the critical Rayleigh number decreases when the inclination angle increases.

\section{References}

[1] S. W. Armfield and J. C. Patterson. Wave properties of natural convection boundary layer. J. Fluid Mech., 239:195-211, 1992. C873

[2] S. W. Armfield and W. Debler. Purging of density stabilized basins. Int. J. Fluid Mech., 36:519-530, 1993. C873

[3] S. W. Armfield. Ellipticity, accuracy, and convergence of the discrete Navier-Stokes equations. J. Comput. Phys., 114:176-184, 1994. C873

[4] S. W. Armfield and R. Janssen. A direct boundary-layer stability analysis of steady-state cavity convection flow. Int. J. Heat Fluid Flow., 17:539 (1996). C877

[5] S. W. Armfield and R. Street. The fractional-step method for the Navier-Stokes equations on staggered grids: the accuracy of three variations. J. Comput. Phys., 153:660-665, 1999. C873

[6] S. S. Cha and K. J. Choi. An interferometric investigation of open cavity natural convection heat transfer. Exp. J. Heat transfer., 2:27-40, 1989. C871

[7] Y. L. Chan and C. L. Tien. A numerical Study of two-dimensional laminar natural convection in shallow open cavities. Int. J. Heat and mass transfer., 28:603-612, 1985. C871 
[8] A. M. Clausing. Convective losses from cavity solar receiverscomparisons between analytical prediction and experimental results. J. Solar Eng., 105:29-33, 1983. C871

[9] A. Javam, J. Imberger and S. W. Armfield. Numerical study of internal wave reflection from sloping boundaries. J. Fluid Mech., 396: 183-201, 1999. C873

[10] B. P. Leonard. A stable and accurate convective modeling procedure based on quadratic upstream interpolation. Comput. Meth. Appl. Mech. Engng., 19:59-98, 1979. C873

[11] J. C. Patterson and S. W. Armfield. Transient features of natural convection in a cavity. J. Fluid Mech., 219:469-498, 1990. C873 